\title{
A Study on Acute Renal Failure in Falciparum Malaria with Special Reference to its Clinical Manifestations, Biochemical Parameters and Management
}

\author{
Manas Kumar Mondal ${ }^{1}$, Arindam Nag ${ }^{2}$, Suraj Mondal ${ }^{3}$, Yashpal Yadav ${ }^{4}$ \\ ${ }^{1}$ Assistant Professor, Department of Gastroenterology, N.R.S Medical College, Kolkata, ${ }^{2}$ Associate Professor, Department \\ of Medicine, R.G. Kar Medical College, Kolkata, West Bengal, ${ }^{3}$ Senior Resident, Department of Medicine, R.G. Kar Medical \\ College, Kolkata, West Bengal, ${ }^{4}$ Senior Resident, Department of Medicine, R.G. Kar Medical College, Kolkata, West Bengal, \\ India
}

Corresponding author: Dr Arindam Nag, 5B Rani Branch Road, P.o.-Paikpara, Kolkata 700002.West Bengal, India

DOI: http://dx.doi.org/10.21276/ijcmsr.2020.5.1.4

BY-NC-ND

How to cite this article: Manas Kumar Mondal, Arindam Nag, Suraj Mondal, Yashpal Yadav. A study on acute renal failure in falciparum malaria with special reference to its clinical manifestations, biochemical parameters and management . International Journal of Contemporary Medicine Surgery and Radiology. 2020;5(1):A16-A20.

\section{A B S T R A C T}

Introduction: Malaria is characterized by intermittent fever with chill and rigor; and hepatosplenomegaly. The involvement of the kidney in falciparum malaria has been known for decades. The initial clinical pattern is that of reversible renal dysfunction or pre-renal azotemia, which rapidly progresses to acute tubular necrosis if treatment is not started.

Material and methods: Fifty cases of acute renal failure selected from amongst 174 diagnosed cases of falciparum malaria admitted in R.G.Kar Medical College \& Hospital were studied (cross sectional study). The cases of acute renal failure were diagnosed on the basis of serum creatinine concentration $>3 \mathrm{mg} / \mathrm{dl}$ ( $>265 \mu \mathrm{mol} / \mathrm{L}$ ) and/or 24-hour urine output < $400 \mathrm{ml}$, despite adequate rehydration, occurring within a few hours to days during the course of the malarial fever. Cases were followed up in the hospital with respect to management and prognosis.

Results: Out of the total of 174 cases of falciparum malaria only 50 cases $(28.7 \%)$ had clinical and biochemical features of renal failure. Analysis of the patients with acute renal failure revealed that pre-renal azotemia secondary to volume depletion or hyper catabolism was present in 14 cases (28\%), and in 36 patients (72\%), the cause of acute renal failure was acute tubular necrosis. Haemodialysis was done in the total $62 \%$ cases. Among 31 patients requiring the haemodialysis 24 (86\%) were oliguric and $07(14 \%)$ non-oliguric.

Conclusion: Cases of pre-renal azotaemia respond well to Antimalarial therapy and conservative treatment with excellent prognosis. Early and frequent Haemodialysis helps in reducing mortality in most of the cases of acute tubular necrosis.

Keywords: MP (Malarial Parsite), PF Antigen (Plasmodium Falciparum), Arf (Acute Renal Failure), Oliguria, Anti Malarial Therapy, Haemodialysis

\section{INTRODUCTION}

The term 'malaria' (derived from Italian word 'Mal' means bad; 'Aria' means air) has been use for over a hundred year to describe the manifestations of a disease caused by a parasite, Plasmodium four species (Plasmodium falciparum, Plasmodium vivax, Plasmodium ovale; and Plasmodium malariae) of which are implicated its aetiology, the disease is being transmitted by the bite of female anopheline mosquito. Malaria characterized by intermittent fever with chill and rigor; and hepatosplenomegaly being its main clinical features. Within the scope of the definition is included "malignant tertian malaria", a potentially fatal form of malaria where blood flow to vital organs like kidney, brain; and lung may be compromised leading to complications like acute renal failure, cerebral malaria; and pulmonary oedema. The involvement of the kidney in falciparum malaria has been known for decades. Multiple factors are involved in the pathogenesis of acute renal failure which has been extensively studied by various workers. The initial clinical pattern is that of reversible renal dysfunction or pre-renal azotemia, which rapidly progresses to acute tubular necrosis if treatment is not started.

Patients with malaria induced renal failure are hypercatabolic with blood urea and serum creatinine levels rising rapidly. ${ }^{1}$ Oliguric as well non-oliguric renal failure are observed and duration of oliguric renal failure ranges from a few days to several weeks depending on the severity of renal dysfunction. Sitprija V. et al. ${ }^{2}$, in one study observed that the overall prevalence of acute renal failure is less than 1\% in falciparum malaria but it occurs in $60 \%$ of patients with severe infection. Acute renal failure in falciparum malaria is usually associated either with acute intravascular haemolysis or heavy parasitemia. $^{3}$

Severe malaria with falciparum infection results in increased sequestration of the red blood cells and consequent 
disturbance of the renal micro-circulation. ${ }^{4}$ Study of the renal cortical blood by Sitprija et al., (1988) ${ }^{4}$ has shown a reduction in cortical perfusion during the acute stage of the disease. Plasma renin activity is also increased indicating reninangiotensin activation in falciparum malaria. the possible role of catecholamine release in the early pathogenesis of renal failure has also been observed. Increased blood viscosity and hypovalemia is also considered contributory to the development of renal failure.

Acute renal failure in falciparum malaria is also observed in patients with severe intravascular haemolysis resulting in haemoglobinuria. It may be induced by malarial fever or by anti-malarial drugs in a patient with or without G6-PD deficiency. The development of renal failure in patients with intravascular haemolysis is attributed to renal ischaemia. Haemoglobin itself is not nephrotoxic, but other compounds released from lysed erythrocytes can induce acute tubular necrosis especially in presence of dehydration and acidosis. Tubular obstruction by haemoglobin casts may also be a contributing factor. ${ }^{5}$

Various other non-specific factors are known to play a role in the pathogenesis of acute renal failure in malaria. Hypovolaemia, jaundice, blood hyperviscosity and intravascular coagulation are critical pre-disposing factors. The association of jaundice with acute renal failure has been studied by various workers. Both haemolytic and cholestatic type of jaundice accompanies the renal failure. Bile acids have been shown to have tubulotoxic effects ${ }^{6}$ and jaundice causes increased vascular sensitivity to catecholamines leading to renal ischaemia. ${ }^{7}$

The mortality rate in acute renal failure is influenced by the severity of oliguria and the presence of other potentially lethal complication. The high incidence of mortality and morbidity associated with acute renal failure has reduced significantly with the advent of modern methods of treatments and availability of dialysis facilities. The prognosis is favourable in patients who have early and frequent dialysis ${ }^{8}$ in their experience of malaria induced renal failure observed that $60 \%$ of patients required dialysis and the mortality decreased from $30 \%$ in the past to less than $10 \%$ at present. Cases with acute renal failure who survive show complete recovery, through minor defects in urine concentrating ability by the renal tubules may persist upto a year.

Purpose of study was to find out the incidence of acute renal failure in falciparum malaria, to study the clinical presentation of ARF in falciparum malaria, to assess efficacy of early anti-malarial treatment in reducing mortality due to ARF in falciparum malaria and to assess the role of dialysis in the outcome of ARF in falciparum malaria.

\section{MATERIAL AND METHODS}

This cross sectional Hospital based study was carried out among the hospitalized patients of R.G.Kar Medical College and Hospital,Kolkata, West Bengal, India during the period from July' 17 to October'18. A total of 50 cases of acute renal failure were selected from patients diagnosed to be suffering from falciparum malaria. Cases of $P$. falciparum malaria, confirmed by detection of P.falciparum antigen and/or in peripheral blood smear (thick and thin films) were taken up for the study. Acute renal failure cases were selected based on the fulfillment of the following basic criteria:

1. Malarial ARF (MARF) is diagnosed when serum creatinine level $>3 \mathrm{mg} / \mathrm{dl}$, and/or urine output $<400$ $\mathrm{ml} / 24 \mathrm{hrs}$ despite adequate rehydration.

2. No past history of renal failure or insufficiency.

3. Normal kidney morphology on USG.

Patients' $\leq 12$ years were excluded from the study.

Members of institutional ethical committee reviewed the protocol of our project titled "A study on acute renal failure in falciparum malaria with special reference to its clinical manifestations, biochemical parameters and management". After deliberations and review, Institutional Ethics Committee took the following decision regarding our project: Approved.

An elaborate history was taken in each patient. Relevant investigations were done like Hb\%, TLC, DLC, ESR, Platelet count, PBS for MP: Thick smear, Thin smear, Pf antigen test: Random blood sugar, L.F.T, PT, LDH, Blood urea, Blood creatinine, Serum $\mathrm{Na}+$ estimation, Serum $\mathrm{K}+$ estimation, Creatinine clearance test, Urinary $\mathrm{Na}+$ estimation (Flame Photometry), ABG analysis, G6PD estimation, $\mathrm{HbsAg}$, Anti HCV,HIV 1\& 2 USG whole abdomen, Chest X-ray - PA view, ECG,Urine examination-Physical - Chemical - Sugar, Albumin, Bile salt, Bile pigment, Phosphate, Microscopic examination.

Each patient with a history of fever, suggestive of severe malaria was subjected to peripheral blood smear examinations, up to 3 samples were examined or till positive and/or Pf antigen positive cases. Patients with falciparum malaria only were considered for the study. Those with plasmodium vivax and mixed infections were excluded. The selected patients were then grouped according to the clinical features, laboratory parameters and the systems involved and were followed up for the outcome. All complicated malaria cases were treated with Quinine as $10 \mathrm{mg} / \mathrm{kg}$ body weight intravenously in $5 \%$ dextrose infused over 4 hrs at 8 hours interval for three days or till the patient becomes conscious and takes Quinine by mouth, whichever is longer. Quinine was given for a total duration of 7 days OR with Artesunate as $2.4 \mathrm{mg} / \mathrm{kg}$ bw IM/IV followed by $2.4 \mathrm{mg} / \mathrm{kg}$ bw after 12 hours then $2.4 \mathrm{mg} / \mathrm{kg}$ bw once daily for total duration of 5 days. All patients were followed with thorough clinical examination and biochemical evaluation as indicated by the patient's condition for the assessment of improvement.

For statistical analysis data were entered into a Microsoft excel spreadsheet and then analyzed by SPSS 24.0. and GraphPad Prism version 5. Data had been summarized as mean and standard deviation for numerical variables and count and percentages for categorical variables. Two-sample $\mathrm{t}$-tests for a difference in mean involved independent samples or unpaired samples. Paired t-tests were a form of blocking and had greater power than unpaired tests. One-way analysis of variance (one-way ANOVA) was a technique used to compare means of three or more samples for numerical data (using the $\mathrm{F}$ distribution). A chi-squared test $\left(\chi^{2}\right.$ test) was any statistical hypothesis test wherein the sampling distribution of the test statistic is a chi-squared distribution when the null hypothesis is true. Without other qualification, 'chi-squared 
test' often is used as short for Pearson's chi-squared test. Unpaired proportions were compared by Chi-square test or Fischer's exact test, as appropriate.

\section{RESULTS}

Out of the 174 patients with severe falciparum malaria, 124 patients (71.3\%) had no renal involvement as evidence by clinical \& biochemical examination and 50 patients $(28.7 \%)$ had acute renal failure in falciparum malaria.

The sex incidence of 50 cases as tabulated above shows that $36(72 \%)$ cases were males and 14 (28\%) were females, indicating a much higher incidence in males.

\begin{tabular}{|l|c|c|c|}
\hline Groups & $\begin{array}{c}\text { No. of } \\
\text { cases } \\
\text { required } \\
\text { dialysis }\end{array}$ & Haemodialysis & $\begin{array}{c}\text { Peritoneal } \\
\text { dialysis }\end{array}$ \\
\hline Severe ATN & 29 & 29 & - \\
\hline Mild ATN & 2 & 2 & - \\
\hline Pre-renal ARF & - & - & - \\
\hline Total & 31 (62\%) & $31(62 \%)$ & - \\
\hline \multicolumn{4}{|c|}{ Table-1: Showing requirement of dialysis in malarial ARF } \\
(MARF).
\end{tabular}

\begin{tabular}{|l|c|c|c|}
\hline Groups & $\begin{array}{c}\text { Cases with } \\
\text { ARF } \\
\text { (ATN) }\end{array}$ & $\begin{array}{c}\text { Cases with } \\
\text { ARF } \\
\text { (Pre-renal) }\end{array}$ & $\begin{array}{c}\text { Total No. of cases } \\
\text { \& Percentage }\end{array}$ \\
\hline No. of cases & 36 & 14 & 50 \\
\hline Survival & $26(72.2 \%)$ & $11(78.6 \%)$ & $37(74 \%)$ \\
\hline Mortality & $10(27.8 \%)$ & $3(21.4 \%)$ & $13(26 \%)$ \\
\hline \multicolumn{4}{|c|}{ Table-2: Showing comparison between development of renal } \\
failure and mortality. \\
\hline
\end{tabular}

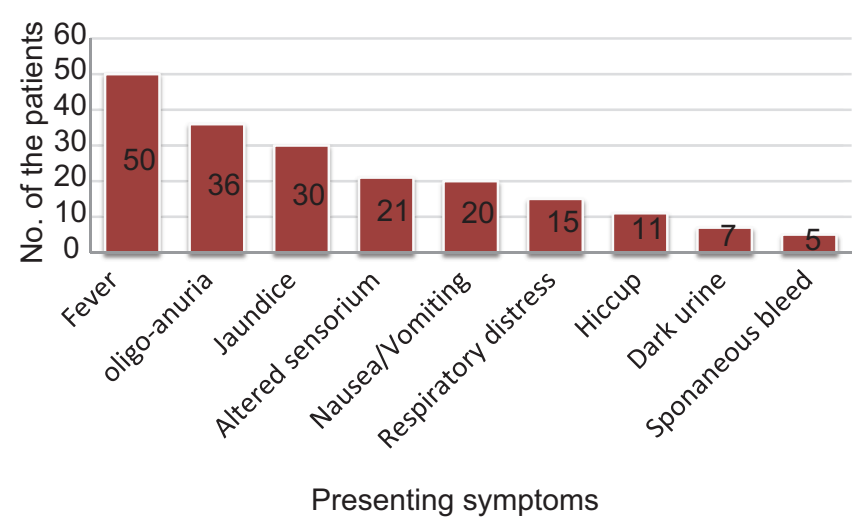

Figure-1: Showing presenting features of the patients

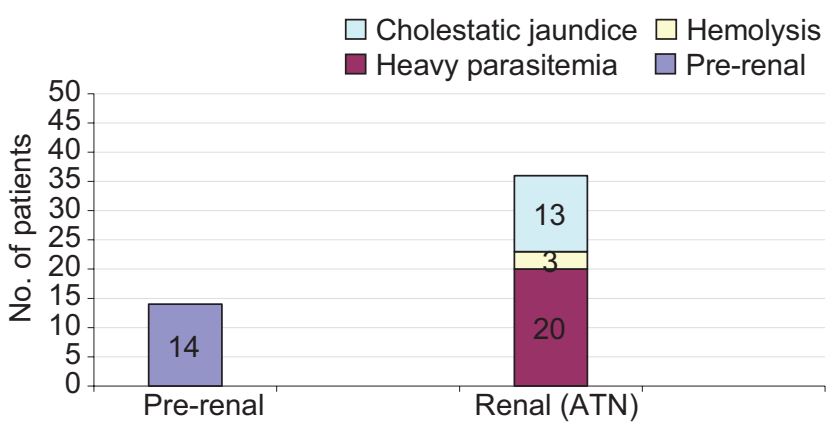

Figure-2: Showing etiology of ARF in falciparum malaria
Approximately $78 \%$ of the cases in the present study were below the age of 40 years. The youngest was 15 years old and the oldest was 61 years old (Mean age $-32 \pm 11.6$ years). Oedema was present in $30 \%$ cases. Mild to moderate hypertension was detected in 16\% and hypotension in $28 \%$ of the falciparum cases. Hepatomegaly \& Splenomegaly were found in $76 \% \& 66 \%$ of the cases respectively. Acidosis was found in $34 \%$ of cases, whereas crackles in the lung were evident in $42 \%$ of the cases.

In the present study majority of the patients $(72 \%)$ were oliguric compared to only $28 \%$ of the patients who were nonoliguric at the time of presentation.

Study of the 24 hours urinary protein excretion revealed that 44 cases (88\%) had increased proteinuria. 14 cases $(28 \%)$ had mild proteinuria in the range of $150-500 \mathrm{mg} / 24 \mathrm{hrs}$, the majority 28 cases (56\%) had proteinuria in the range of 500$1000 \mathrm{mg} / 24 \mathrm{hrs}$. Among them 2 patients (4\%) had increased protein excretion greater than $1000 \mathrm{mg} / 24 \mathrm{hrs}$.

Out of the 50 patients 45 had marked reduction in GFR with a mean value of 9.68 , whereas other 5 patients had less marked fall of GFR with a mean value of 25.59 .

Study of the renal function tests in the above table reveals that majority of the patients had significant rise in blood urea level with a mean value of $177 \mathrm{mg} \%$. S.creatinine levels ranged between $3.2-13.6 \mathrm{mg} \%$ with a mean value of 7.83 $\mathrm{mg} \%$. The mean creatinine clearance rate was $11.71 \mathrm{ml} / \mathrm{min}$. Out of the 36 cases of acute tubular necrosis, 29 cases were found to have severe ATN and 7 cases had milder form of ATN based on the serum creatinine levels, GFR and recovery of renal function.

Patients presented with oliguric renal failure, Oliguric phase lasted for approximately 8-15 days (Mean-12) in the severe form of renal failure. Whereas, duration of oliguria in the mild form of renal failure was 4-7 days (Mean-5). 58\% patients received quinine $\& 42 \%$ received artisunate in acute renal failure due to falciparum malaria.

\section{DISCUSSION}

In a similar study by Sitprija et al., (1970), ${ }^{4}$ acute renal failure was noted in $21 \%$ of the cases. In other studies by Bouth DM. Giboda M (1987), and Weber, Horstmann ${ }^{9}$ (1991), an incidence of $23.3 \%$ and $29 \%$ respectively were detected. Habte et al. ${ }^{10}$, (1992) observed a slightly higher incidence of $33.3 \%$ in their study with twenty three patients. All these results are more or less similar to the present observation. Mishra SK et $\mathrm{al}^{11}$., (1992) has reported renal dysfunction in $33 \%$ of the patients in falciparum malaria. This is in contrast with the much lower incidence (13\%) reported by Sharma AK et al. $.^{12},(1998)$ in North India. Padhi PK et a ${ }^{13}$., (2003) and Krishnan A et a ${ }^{14}$., (2003) have reported ARF in 29.4\% and $30.23 \%$ of the cases respectively.

Stone et al. ${ }^{1}$, (1972) have reported $85 \%$ oliguric renal failure. Habte B et $\mathrm{al}^{10}$. ., (1990) in their study of 72 patients with severe falciparum malaria, observed oliguric renal failure in $45 \%$ of cases. Wilairatana P et al..$^{15}$, (1999) have reported 70\% oliguric acute renal failure in falciparum malaria. Similarly, Junejo Abdul Manan et al. ${ }^{16}$ (2006) was seen in $76.09 \%$ cases of falciparum malaria on admission.

Non-oliguric renal failure has also been recognised by other 
workers. ${ }^{17} \mathrm{~A}$ fewer incidence of complications and a better prognosis associated with non-oliguric renal failure has been observed in various studies by other workers. A fatal outcome was associated significantly with anuria. ${ }^{18}$ In a study by Sitprija V et al., (1970) 4 on patients with malaria induced renal failure, pre-renal azotaemia was detected in $40 \%$ of the patients and acute tubular necrosis developed in $60 \%$ cases. A comparatively higher incidence of acute tubular necrosis of $72 \%$ in the present study is attributed to the fact that most of the patients were from rural background and were admitted to the hospital rather late during the course of the illness. The initial clinical presentation in malaria induced renal failure is that of reversible renal dysfunction or pre-renal form, and if renal ischaemia is prolonged, it rapidly progresses to acute tubular necrosis (fig-1). Sitprija V et al., (1970) ${ }^{4}$ in their study of 18 patients with acute tubular necrosis observed that severe intravascular haemolysis was the cause of acute tubular necrosis in 1 patient (5.6\%), while in the majority $94.4 \%$ of the patients, renal failure was attributed to heavy parasitaemia. Mahakur AC et al. ${ }^{19}$ (1987) \& J. Prakash et al..$^{20}$ (1996) observed ARF due to hyperparasitaemia in 30.8\% of cases, intravascular haemolysis in $30.8 \%$, and cholestatic jaundice in $23 \%$ of cases.

So, the etiology of the ATN in the present study almost similar with the above mentioned studies (Figure 2). In a study by Stone et al., ${ }^{1} 85.6 \%$ were oliguric and $14.4 \%$ were not. Although both decreased glomerular filtration rate \& tubular obstruction contribute to the development of oliguria in acute renal failure. In falciparum malarial nephropathy, a renal haemodynamic alteration plays the major role. ${ }^{21}$ Trang TT, MJ White et al., $1992^{18}$ in their study of sixty four patients with malaria induced renal failure, observed that recovery of renal function was unrelated to parasitaemia or haemoglobinuria and the mean duration for serum creatinine levels to return to normal was 17 days. In the patients with milder form of acute tubular necrosis, mean value of serum creatinine was $4.8 \mathrm{mg} / \mathrm{dl}$ and GFR of $12.7 \mathrm{ml} / \mathrm{min}$, whereas in patients with severe form, $\mathrm{S}$. creatinine showed a mean value of $14.6 \mathrm{mg} \%$. Recovery of renal function was delayed in the severe form ranging between 21 to 22 days, whereas it took approximately 9 to 15 days for $\mathrm{S}$. creatinine to return to normal in the milder form. J Prakash et al. ${ }^{22}$ (2002) was used Quinine in majority $78.7 \%$ of patients and remaining $21.3 \%$ cases were treated with artimisinine derivatives. In this study Quinine was used more number of patients than the present study. Similar observation has been made by Stone et al. ${ }^{1}$, (1972) and Weber MW et $\mathrm{al}^{9}$., (1991) in their study of malaria induced renal failure. Dialysis was required in $69.2 \%$ and $70 \%$ of their cases respectively.

Haemodialysis is preferred in severe acute renal failure caused by falciparum malaria. Peritoneal dialysis is less effective under most circumstances because of impaired peritoneal microcirculation due to parasitized erythrocytes and vasoconstriction which results in reduced solute transport. ${ }^{4}$ Habte B. et al., ${ }^{10}$ (1990) observed a mortality rate of $29 \%$ from their study of twenty four patients with malaria induced renal failure.

Junejo Abdul Manan et al. ${ }^{16}$ (2006) in their study of malaria induced renal failure showed that $88.89 \%$ were oliguric and
11.11\% non-oliguric renal failure required Haemodialysis and also they measured the average number of dialysis sessions required per patient was 06 , with a minimum of 03 to a maximum of 15 . The oliguric patients also needed more Haemodialysis sessions than the non-oliguric patients (Table 1)

In another study, Sitprija et al. ${ }^{4}, 1988$ observed a decrease in the mortality rate from $30 \%$ in the past to less than $10 \%$, while Stone et al., in their study of 42 cases of malarial ARF reported a mortality rate of $28.9 \%$. These findings are consistent with the present findings. (Table 2)

\section{CONCLUSION}

A sizable number of cases of falciparum malaria develop acute renal failure in the form of pre-renal azotemia and acute tubular necrosis. Cases of pre-renal azotaemia respond well to Antimalarial therapy and conservative treatment with excellent prognosis. Early and frequent Haemodialysis helps in reducing mortality in most of the cases of acute tubular necrosis. Severity of oliguria and presence of one or more associated complications like pulmonary oedema, acidosis, and altered sensorium have considerable influence on the outcome of the patients. Hence, it is essential to look for these ominous parameters which have adverse outcome in renal failure associated with falciparum malaria. As the study was conducted with small number of cases, further study is necessary with large number of cases to arrive at a conclusion regarding the malady of acute renal failure in falciparum malaria.

\section{REFERENCES}

1. Stone WJ, Hanchett JE, Knepshield JH. Acute renal failure due to falciparum malaria. Review of 42 cases. Arch Intern Med 1972; 129(1): 620-8.

2. Sitprija V, Indraprasit S, Pochanugool C, Benyaiati C, Piyaratn P. Renal failure in malaria. Lancet 1967; 1(5): 185-88.

3. Eiam-Ong S, Sitprija V. Falciparum malaria and the kidney: a model of inflammation. Am J Kidney Dis 1998; 32(3): 361-75.

4. Sitprija V. Nephrology forum. Nephropathy in falciparum malaria. Kidney Int 1988; 34(2): 966.141-7.

5. Rosen S, Hano JE, Inman MM et al. The kidney in black water fever. Am J Clin Pathology 1968; 49(4): 358-70.

6. Aoyagi T, Lowenstem LM. The effect of bile acid and renal ischaemia on renal function. J Lab Clin Med 1969; 71(6): 686-690.

7. Bloom D, McCalden TA, Rosendroff C. Effect of jaundiced plasma on vascular sensitivity to noradrenalin. Kidney Int 1975; 8(5): 149-57.

8. Sitpnja V.Vongsthongsni M,Poshyachinda V.Arthacinta 5, 1977. Renal failure in malaria: a pathophysiologic study. Nephron 18(3): 277 - 287.

9. Weber MW, Boker K, Horstmann RD, Ehrich JH. Renal failure is a common complication in non-immune Europeans with Plasmodium falciparum malaria. Trop Med Parasitol 1991; 42(2): 115-118.

10. Habte B. Acute renal failure due to falciparum malaria. Renal fail 1990; 12(5): 15-19.

11. Mishra SK, Mohanty B, Das BS, et al., - Hepatic 
changes in falciparum malaria. Indian J Malariol 1992; 29(1): I 67-71.

12. Sharma AK, Arora M, Gupta H, Gupta R. Malarial Acute renal failure in Rajasthan. J Assoc Physician India 1998; 46(3):1001-1002.

13. Padhi PK, Srikant KV, Devi A, Increasing incidence of complicated Falciparum malaria and its varied manifestations. JAPI: 2003, Jan; 51.

14. Krishnan A, Karmad DR et al. severe falciparum malaria Crit Care Med 2003; 31(6):2278-84.

15. Wilairatana P, Westerlund EK, Aursudkij B, Vannaphan S, Krudsood S, Viriyavejakul P, et al. Treatment of malarial acute renal failure by hemodialysis. Am J.Trop. Med. Hyg 1999; 60(2): 233-37.

16. Junejo Abdul Manan, Hassan Ali, Manohar Lal, Acute renal failure associated with malaria.J Ayub Med Coll Abbottabad 2006; 18(4).

17. Sitprija V. Nephrology forum. Nephropathy in falciparum malaria. Kidney Int 1988; 34(1): 966.141-7.

18. Trang TT, Phu NH, Vinh H, Hien TT, Cuong BM, Chau TT, et al. Acute renal failure in patients with severe falciparum malaria. Clin Infect Dis 1992; 15(6):874-80.

19. Mahakur AC, Baliarsinha A, Nanda BK, Bose TK, Sapathy SR, Mishra Y. Prevalence and pattern of renal lesions in plasmodium falciparum malaria. Ind J Nephrol 1987; 3(5): 66-73.

20. J.Prakash, A. Gupta1, O. Kumar, S. B. Rout, V. Malhotra and P. K. Srivastava. Acute renal failure in Falciparum malaria-increasing prevalence in some areas of India- a need for awareness. Nephrol Dial Transplant 1996;11(1): 2414-2416.

21. Lumlertgul D, Keoplung M, Sitprija V, Moollaor P, Suwangool P. Furosemide and dopamine in malarial acute renal failure. Nephron 1989; 52(6): 40-4.

22. J Prakash, AK Singh, S Gujrati, A Maheshwari. Acute renal failure in Malaria: Changing trends. Indian J Nephrol 2002; 12(6): 113-117.

Source of Support: Nil; Conflict of Interest: None

Submitted: 02-12-2019; Accepted: 23-12-2019; Published online: 17-01-2020 